\title{
Rapid Assessment of Anthocyanins Content of Onion Waste through Visible-Near-Short-Wave and Mid-Infrared Spectroscopy Combined with Machine Learning Techniques
}

\author{
Nikolaos Tziolas ${ }^{1}{ }^{(}$, Stella A. Ordoudi ${ }^{2}{ }^{\circledR}$, Apostolos Tavlaridis ${ }^{3}$, Konstantinos Karyotis ${ }^{1}$, George Zalidis ${ }^{1}$ and \\ Ioannis Mourtzinos ${ }^{3, *(D)}$ \\ 1 Laboratory of Remote Sensing, Spectroscopy, and GIS, Department of Agriculture, Aristotle University of \\ Thessaloniki, 54124 Thessaloniki, Greece; ntziolas@agro.auth.gr (N.T.); kkaryotis@ihu.edu.gr (K.K.); \\ zalidis@agro.auth.gr (G.Z.) \\ 2 Laboratory of Food Chemistry and Technology, School of Chemistry, Aristotle University of Thessaloniki, \\ 54124 Thessaloniki, Greece; steord@chem.auth.gr \\ 3 Department of Food Science and Technology, School of Agriculture, Aristotle University of Thessaloniki, \\ 54124 Thessaloniki, Greece; tolistavl@gmail.com \\ * Correspondence: mourtzinos@agro.auth.gr; Tel.: +302-310-991-637
}

\section{check for}

updates

Citation: Tziolas, N.; Ordoudi, S.A.; Tavlaridis, A.; Karyotis, K.; Zalidis,

G.; Mourtzinos, I. Rapid Assessment of Anthocyanins Content of Onion Waste through Visible-Near-ShortWave and Mid-Infrared Spectroscopy Combined with Machine Learning Techniques. Sustainability 2021, 13, 6588. https://doi.org/10.3390/ su13126588

Academic Editor: Michael S. Carolan

Received: 12 May 2021

Accepted: 5 June 2021

Published: 9 June 2021

Publisher's Note: MDPI stays neutral with regard to jurisdictional claims in published maps and institutional affiliations.

Copyright: (c) 2021 by the authors. Licensee MDPI, Basel, Switzerland. This article is an open access article distributed under the terms and conditions of the Creative Commons Attribution (CC BY) license (https:// creativecommons.org/licenses/by/ $4.0 /)$.

\begin{abstract}
A sustainable process for valorization of onion waste would need to entail preliminary sorting out of exhausted or suboptimal material as part of decision-making. In the present study, an approach for monitoring red onion skin (OS) phenolic composition was investigated through Visible Near-Short-Wave infrared (VNIR-SWIR) (350-2500 nm) and Fourier-Transform-Mid-Infrared (FT-MIR) (4000-600 cm ${ }^{-1}$ ) spectral analyses and Machine-Learning (ML) methods. Our stepwise approach consisted of: (i) chemical analyses to obtain reference values for Total Phenolic Content (TPC) and Total Monomeric Anthocyanin Content (TAC); (ii) spectroscopic analysis and creation of OS spectral libraries; (iii) generation of calibration and validation datasets; (iv) spectral exploratory analysis and regression modeling via several ML algorithms; and (v) model performance evaluation. Among all, the k-nearest neighbors model from 1st derivative VNIR-SWIR spectra at 350-2500 nm resulted promising for the prediction of TAC $\left(R^{2}=0.82\right.$, RMSE $=0.52$ and RPIQ $\left.=3.56\right)$. The 2nd derivative FT-MIR spectral fingerprint among $600-900$ and $1500-1600 \mathrm{~cm}^{-1}$ proved more informative about the inherent phenolic composition of OS. Overall, the diagnostic value and predictive accuracy of our spectral data support the perspective of employing non-destructive spectroscopic tools in real-time quality control of onion waste.
\end{abstract}

Keywords: VNIR-SWIR; FT-MIR; chemometrics; onion solid waste; natural colorant

\section{Introduction}

Huge amounts of onion (Allium cepa L) waste, consisting mainly of the skin and inedible outer scales of the bulb, are generated throughout their supply chain from the farm to retail stores and the households. In 2000, more than 450000 tonnes of onion solid waste (OSW) were produced in Europe [1]; the tonnage is expected to be much higher today with increasing production [2]. OSW can be considered as an environmental problem because it is not suitable for use as organic fertilizer due to the rapid development of phytopathogenic agents, or as a fodder because of its aroma [3].

A possible solution could be the development of a sustainable process to convert this food waste into a raw material for the food industry [1,4]. Regardless of the season, cultivar, or ripening stage, OSW can be a potential source of fibers, fructooligosaccharides, the alk(en)yl cysteine sulfoxides and certain health-promoting phenolic compounds, especially flavonoids [3]. Onion flavonoids are found especially in the skin and outer layers [5], mainly in the form of quercetin aglycone. At least two major types (including quercetin- 
3,4'-O-diglucoside (3,4'-Qdg) and quercetin-4'-O-glucoside (4'-Qmg) [3]) conjugate with glucose.

OSW from red-skinned onions is also rich in anthocyanins [6]. Even though anthocyanins comprise a small percentage of onion bulb flavonoids, they are heavily concentrated in the skin and in a single layer of cells in the epidermal tissue, mainly in the form of cyanidin glucosides, esterified with malonic acid [6]. Red onion dry outer layers could, therefore, be a source of natural colorants that can be extracted with green extraction techniques and used as a replacement of synthetic counterparts like carmine [7].

Several novel technological solutions have been proposed to recover these valuable ingredients from OSW [8]. Many studies focus on improving the incorporation of the extracts into novel, biofunctional food products or in the formulation of food supplements $[9,10]$. The overall cost/sustainability of the biorefinery processes depend on various factors $[1,11]$ that must comply with rational strategies for waste management, such as stabilization and quality. Standards for preliminary sorting to exclude exhausted or sub-optimal waste would be of great value.

Onsite diagnostic assessment of the onion waste content in phenolic compounds is quite challenging. Complexity of supply chains, multi-scaled production, and heterogeneity of the waste composition hinder large-scale operational investigations. Electrochemical sensors are already used in the food industry as sensitive tools for monitoring polyphenol content in certain commodities [12]. Studies show that non-destructive spectroscopic techniques in the visible-near-short-wave and mid-infrared regions combined with powerful chemometric methods may offer cost-effective, rapid, and versatile tools for monitoring the chemical composition of foods. In this context, the use of VNIR-SWIR and FT-MIR spectrometers at all relevant stages across the onion supply chains along with implementation for quality control of thousands of OSW samples generated would inform decision-making about further waste management processes (re-use, re-cycle, valorization etc.). Whether such technology is mature enough for application to routine analysis of OSW is open to question. Artificial intelligence through machine learning (ML) algorithms has revolutionized the predictive performance of current chemometric methods used in the food sector [13]. In the case of onions, a partial least square regression (PLSR) model for the assessment of total phenolic content and total antioxidant activity of phenolic-rich extracts of onion bulbs has been reported to fit well with data extracted from FT-IR spectral features [14]. However, systematic exploitation of spectral analysis and ML algorithms in OSW lags greatly behind. Some years ago, Vincke et al. [15] utilized NIR spectroscopy along with a Partial Least-Squares Discriminant Analysis (PLS-DA) to automatically sort different parts of onion bulbs produced during specific industrial processes. At that time, Wang and Gitaitis [16] highlighted that light-scattering properties of different onion parts in the VNIR region may be further exploited for non-destructive inspection of diseased onions, but they did not employ chemometric tools in their investigation.

The overarching objective of this study was to examine whether chemometric modelling of VNIR-SWIR spectral data of red OS powder, according to their actual content in monomeric anthocyanins, can feasibly be used for predictive purposes. Reference Ultraviolet-visible (UV-Vis) based chemical assays were employed as a first step to assess the content in total phenols and monomeric anthocyanins. Having specified how the spectral signatures in the VNIR-SWIR were to be recorded under standard acquisition protocols, a series of state-of-the-art ML algorithms for regression analysis were deployed using the whole or sub-regions of the VNIR-SWIR spectra as variable inputs. Whether the diagnostic spectral region for anthocyanins might be extended to the mid-infrared spectrum by employing FT-MIR spectroscopy (in attenuated total reflectance (ATR) mode) was also investigated. In that case, an unsupervised ML-based exploratory approach was used to search for interpretable patterns among the OS spectra.

The focus of this study was to document the steps, driven by the current findings, to turn infrared spectroscopy into an operational tool for the assessment of the anthocyanins present in OSW. 


\section{Materials and Methods}

The methodological approach consists of five discrete steps: (i) chemical analysis to obtain reference values for Total Phenolic Content (TPC) and Total monomeric anthocyanins (TAC); (ii) spectroscopic analysis (VNIR-SWIR/ATR-FT-MIR), which includes the creation of the dry OS spectral libraries; iii) generation of calibration and validation datasets; (iv) spectral exploratory analysis and regression modeling of VNIR-SWIR spectra where several ML algorithms were evaluated to predict the content of anthocyanins; and (v) evaluation of the performance metrics obtained by ML algorithms. The overall data processing and analysis workflow is illustrated in Figure 1 and detailed descriptions of the different steps are provided in the sections below.

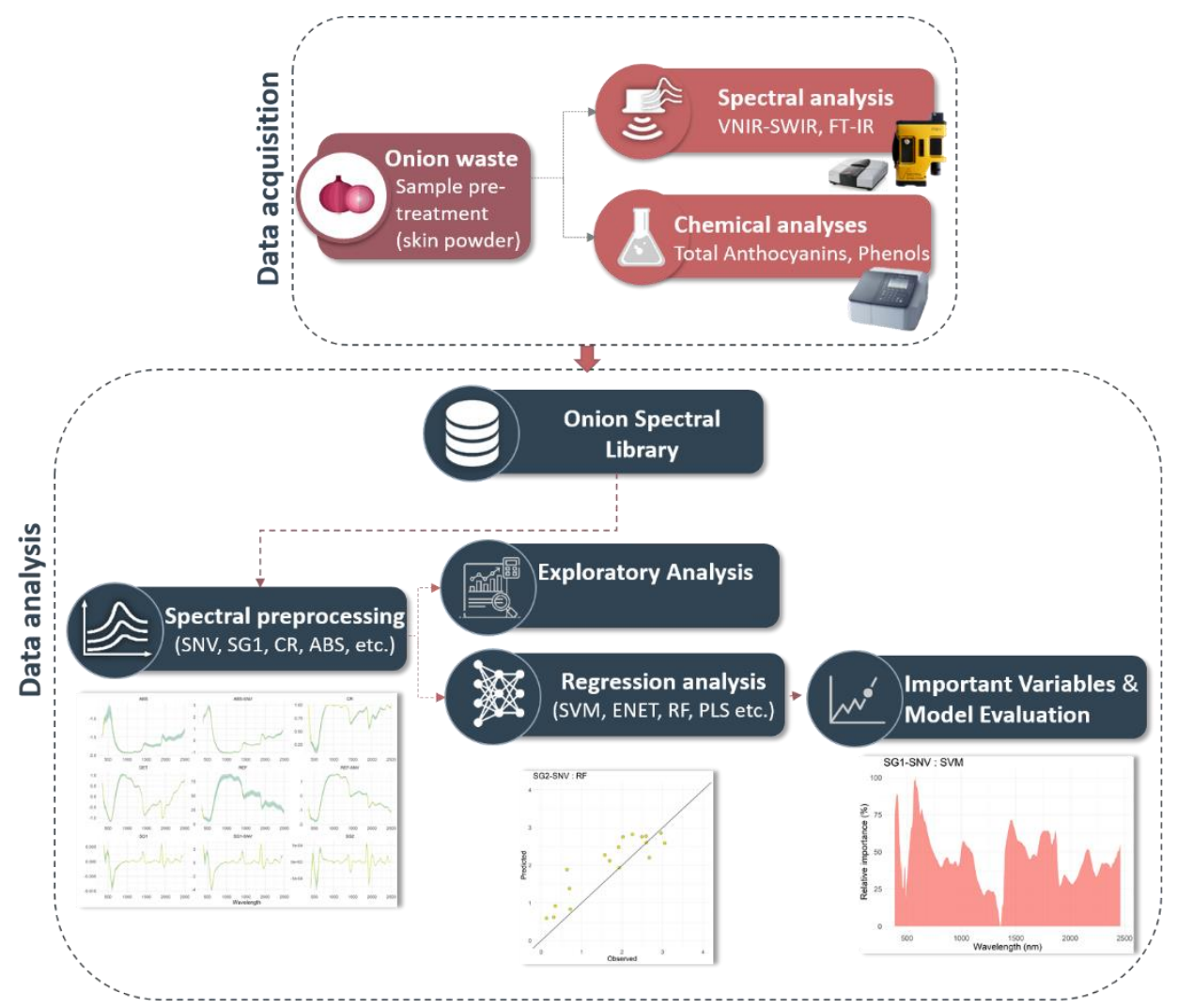

Figure 1. Flowchart of the proposed workflow for the onion skin spectral analysis.

\subsection{Onion Samples (OS)}

The bulbs of the Allium cepa L. were supplied from various local markets in Greece during the autumn-winter season of 2019 to represent three different retail product lines: one originating from the Netherlands $(n=25)$ and two from major producing regions in Greece (Thiva, Evritania, $\mathrm{n}=13$ ).

The onion skin (OS) test samples were prepared as follows. The bulbs of the red onions were peeled using a sharp blade to remove the outer dry layers and the apical trimmings, which are considered as waste material. Different layers of each sample (dry outer, first inner) were separated. The dry outer and first inner layer skins of each bulb were washed twice in deionized water. The resulting materials were dried at $65^{\circ} \mathrm{C}$ for $48 \mathrm{~h}$, ground using a domestic blender (KENWOOD, Havant, UK), powdered in a laboratory mill and then sieved through a $0.5 \mathrm{~mm}$ mesh. The material was then mixed to represent 38 test samples of distinct origin. Random combinations of 6 out 38 samples were produced $(n=8)$ to enhance color variance. The inner and outer layers of 15 individual bulbs from the Netherlands batch were also treated separately $(n=26)$. In total, 72 OS test samples were used in this study. 


\subsection{Chemical Characterization of the OS Extract}

\subsubsection{Chemicals}

All solvents or analytical standards, such as Folin-Ciocalteu reagent, gallic acid, and sodium chloride $\left(\mathrm{Na}_{2} \mathrm{CO}_{3}\right)$, were purchased from Sigma-Aldrich, Chemie $\mathrm{GmbH}$ (Taufkirchen, Germany).

\subsubsection{Preparation of OS Extract}

OS powder was mixed with solvent (liquid-to-solid ratio of $10 \mathrm{~mL} / \mathrm{g}$ ), composed of $(70 \% v / v)$ ethanol in water, at $\mathrm{pH}=1$. The material was subjected to extraction at $25^{\circ} \mathrm{C}$ for $15 \mathrm{~min}$ in an Ultrasons-H ultrasonic bath (J.P. Selecta Barcelona, Spain). Following extraction, the samples were filtered through a $0.45 \mu \mathrm{m}$ nylon membrane filters (BGB, USA). The clear supernatant was stored at $-20^{\circ} \mathrm{C}$ until used for further analysis.

\subsubsection{Determination of Total Phenolic Content (TPC)}

In brief, $30 \mu \mathrm{L}$ of all dissolved extracts were mixed, separately, with $2370 \mu \mathrm{L}$ of deionized water and $150 \mu \mathrm{L}$ undiluted Folin Ciocalteu's reagent. After one minute, $450 \mu \mathrm{L}$ $\mathrm{Na}_{2} \mathrm{CO}_{3}(20 \%, w / v)$ was added. The mixture was incubated for $120 \mathrm{~min}$ and absorbance of the resulting mixture was measured spectrophotometrically at $750 \mathrm{~nm}$. Gallic acid was used as a reference standard and the results were expressed as milligram gallic acid equivalents (mg GAE)/g of extract.

\subsubsection{Determination of Total Monomeric Anthocyanin Content (TAC)}

TAC was determined according to [17] using the pH-differential method. Briefly, absorbance readings at $510 \mathrm{~nm}$ and $700 \mathrm{~nm}$ were made after dilution of extract in buffers solution, with $\mathrm{pH}$ values of 1.0 and 4.5, against distilled water. The calculation was based on Equations (1) and (2), respectively:

$$
A=\left(A_{\lambda \max }-A_{700}\right)_{p H 1.0}-\left(A_{\lambda \max }-A_{700}\right)_{p H 4.5}
$$

where $A \lambda \max$ is the absorbance of the sample extract at $510 \mathrm{~nm}$

$$
\text { Total monomeric anthocyanins }\left(\frac{m g}{100 g}\right)=A \times M_{w} \times D f \times \frac{1000}{\varepsilon \times l}
$$

where $M_{w}$ (molecular weight $)=449.2 \mathrm{~g} /$ mole for cyanidin-3-glucoside; $D f=$ dilution factor; $l=$ pathlength in $\mathrm{cm} ; \varepsilon=26,900$ molar extinction coefficient in $L \times$ mole $^{-1} \times \mathrm{cm}^{-1}$ for cyanidin-3-glucoside; $10^{3}=$ conversion of $\mathrm{g}$ to $\mathrm{mg}$. The results are expressed as $\mathrm{mg}$ cyanidin-3-glucoside per $100 \mathrm{~g}$ of onion dry matter. All analyses were performed in triplicate and the median values were calculated. The summary statistics of the chemical analyses are presented in Table 1. In the table below, Q1, Q2, and Q3 denote the quartiles. Q1 corresponds to the lowest $25 \%$ of numbers, Q2 ranges between $25.1 \%$ and $50 \%$ (up to the median), and Q3 corresponds to the range $50.1 \%$ to $75 \%$ (above the median).

Table 1. Summary statistics of Total Phenolic (TPC) and Total Monomeric Anthocyanin Content (TAC) values of the OS samples under study $(n=72)$.

\begin{tabular}{ccccccc}
\hline Parameter & Min & Max & Q1 & Q2 & Q3 & Mean \\
\hline TPC $(\mathrm{mg}$ GAE $/ \mathrm{g})$ & 13 & 79 & 30 & 43 & 52 & 43 \\
TAC $($ mg Cyanidin/g) & 0.13 & 3.82 & 1.49 & 2.08 & 2.71 & 2.01 \\
\hline
\end{tabular}

\subsection{Spectroscopic Characterization of the Dry OS}

In this section, we briefly present the methodological steps to develop an onion spectral library that would be used for assessing the total anthocyanin content. The building of a database for OS anthocyanins that utilizes their unique spectral signatures (combination of infrared bands) in specific spectral regions is described. 


\subsubsection{VNIR-SWIR Analysis}

The VNIR-SWIR measurements of dry red OS powdered samples were performed using a PSR +3500 spectrometer (Spectral Evolution Inc., Lawrence, Massachusetts, USA) operating in the range 350 to $2500 \mathrm{~nm}$. The measurements were performed using a contact probe to eliminate the effects of light scattering. Five spectra per powdered sample were recorded and averaged to obtain the corresponding reflectance spectral signatures. A Spectralon ${ }^{\circledR}$ panel with $99 \%$ reflectance was used to calibrate the spectrometer before the measurements.

\section{Spectral Preprocessing Techniques}

Widely employed scatter-corrective and spectral-derivatization preprocessing techniques were applied to the VNIR-SWIR dataset to remove irrelevant information. In brief, (i) the reflectance spectra (REF) were converted into (ii) pseudo-absorbance spectra $[\log 10(1 / R)](A B S)$, and (iii) transformed into a continuum removal method domain (CR). The Standard Normal Variate (SNV) was then applied to both REF and ABS values resulting to (iv) REF-SNV and (v) ABS-SNV datasets, respectively. The Savitzky-Golay method was applied to remove unwanted background noise from the spectra (vi) by calculating the first derivative (SG1), and in that case, (vii) combining with the SNV transformation (SG1SNV) and also (viii) by calculating the second derivative (SG2), 11 data points of interval. Lastly, (ix) the detrend (DET) preprocessing method was used before data modelling. An overview of these techniques is presented by Rinnan et al. [18]. In total, nine different spectral datasets were produced.

\section{Machine Learning Modeling}

The Conditioned Latin hypercube method (cLHS) [19] was used to split the onion VNIR-SWIR spectral data into calibration and validation datasets. According to the cLHS algorithm, the method searches the data based on heuristic rules combined with an annealing schedule. The proposed method is considered to be an effective way of replicating the distribution of the variables compared to a random sampling approach. The percentage of the number of onion samples to be allocated for the calibration dataset was determined as $75 \%$ of total dataset (54 out of 72 ), while the rest (18) were included in the validation set.

Each dataset of preprocessed spectra was modelled against TAC values using the following linear or non-linear regression algorithms, i.e., (i) partial least square regression (PLS); (ii) Random Forest (RF); (iii) Cubist; (iv) elastic net (ENET); (v) k-nearest neighbors (k-NN); and (vi) support vector machines for regression (SVM). In every method, a set of hyperparameters was selected as follows. The classical PLS algorithm [20], widely applied for multiple purposes in spectroscopic analysis, transforms the input factors' matrix into a series of latent variables (LVs) to maximize the covariance among the predictors and dependent variables. The number of optimum LVs was selected to range from 10 to 30 . $\mathrm{RF}$ is an ensemble learning classifier [21] with good performance metrics in various spectroscopy studies. Tuning of hyperparameters included first the selection of a number of variables that can be sampled in each split of the tree analysis $(6,24)$ and then the value of the tree parameter $(100,250,500,1000,1500)$. The rule-based Cubist algorithm [22] reduces a set of rules derived by a decision tree to define a linear regression model. Then, multiple rule-based models are combined (committees) and the final predictions are adjusted using known errors on the training set with a small number of neighbors for each unknown sample. Thus, predefined values for the number of committees $(1,10,50,100)$ and of neighbors $(0,1,5,9)$ were selected. The ENET method was also evaluated as an extension of linear regression that adds regularization penalties to the loss function during training. Next, the $k$-NN algorithm was used. This is an instance-based learning algorithm that utilizes a distance metric from the calibration dataset and predicts a testing pattern depending on a preselected number, $k$, of nearest neighbors. In our study, this value was optimized in a range from 0 to 25 closest k neighbors. Lastly, SVM for regression, as introduced by Drucker et al. [23], was evaluated. SVM is a non-parametric technique employing a kernel 
function to map the initial predictors into a higher dimensional space. In this study, a radial basis function was utilized, while the $C$ parameter values were optimized among $(0.001$, $0.01,1,10)$ to control the penalization of the residual errors.

A grid search on a five-fold cross-validation experiment for each analysis enabled the selection of the optimal hyperparameter values for model consistency. Table 1 in Appendix A shows the optimal hyperparameter values for each ML algorithm. In total, 54 calibration models were produced. In order to assess their performance for prediction of TAC in dry red OS, the root-mean-square error (RMSE, Equation (3)), the coefficient of determination ( $\mathrm{R}^{2}$, Equation (4)), and the Ratio of Performance to Interquartile Range (RPIQ; Equation (5)) values were compared. The equations used were as follows:

$$
\begin{gathered}
R M S E=\sqrt{\frac{\sum_{i=1}^{i=N}\left(y_{i}-\hat{y}_{i}\right)^{2}}{N}} \\
R^{2}=1-\frac{\sum_{i=1}^{i=N}\left(y_{i}-\hat{y}_{i}\right)^{2}}{\sum_{i=1}^{i=N}\left(y_{i}-\bar{y}\right)^{2}} \\
R P I Q=\frac{I Q}{R M S E}
\end{gathered}
$$

where $y_{i}$ is the observed value and $\hat{y}_{i}$ is the predicted value of sample $i, N$ is the number of observations (Equation (3)), $\mathrm{y}$ is the mean of the observed values (Equation (4)), and IQ is the interquartile range (IQ = Q3 - Q1) of the observed values (Equation (5)). Q1 and Q3 denote the first and third quartile, respectively.

\subsubsection{ATR-FT-MIR Analysis}

ATR-FT-MIR spectra were acquired using a 6700 IR (Jasco, Essex, UK) spectrometer equipped with a DLaTGS detector, a high-throughput Single Reflection ATR with diamond crystal and complemented by the Spectra Manager software (Jasco, Essex, UK). For each spectrum, eight scans were accumulated in the absorbance mode and recorded at $4 \mathrm{~cm}^{-1}$ resolution, covering a range from 4000 to $600 \mathrm{~cm}^{-1}$. The spectrum was collected against a background obtained with a dry and clean cell and corrected by the ATR correction option of the software. Three spectra per powdered sample were recorded and averaged to obtain the corresponding spectrum before further preprocessing.

Spectral artifacts due to noise, baseline offset, and slope or light scattering were removed by the multiplicative signal correction method (MSC) and second order derivatization with the Savitzky-Golay method (11 data points of interval) [24]. The spectral data were mean-centered and further processed via Principal Component Analysis (PCA). PCA is an unsupervised technique that transforms a set of variables into a new set of composite variables, the principal components (PCs). PCA attempts to simplify the distribution of samples and identify the underlying factors that explain possible patterns of variable and sample correlations. For exploratory purposes, only principal components with eigenvalue $>1.0$ were considered useful, according to the Kaiser criterion [25].

\subsection{Implementation}

The statistical and regression analyses of the VNIR-SWIR datasets were performed utilizing the R programming language [26], with the caret package [27]. The commercial SIMCA 16.02 software (Umetrics, Sweden) was used for FT-MIR spectral analysis.

\section{Results and Discussion}

\subsection{Chemical Analyses Data}

The phenolic constituents of red onion skin are expected to exist primarily in bound form [5]. Nevertheless, the TPC values of red OS samples that represent mainly free soluble forms of phenolic compounds were found to range between 13 and $79 \mathrm{mg} \mathrm{GAE} / \mathrm{g}$. These values fall within typical ranges for the outer layers of brown-skin onion bulbs that have 
previously been reported in literature $[5,28]$, regardless of the geographical origin of the bulb or the extraction method.

The soluble phenolic extracts of OS samples were found to be rich in anthocyanins. In particular, the TAC values varied between 0.13 and $3.82 \mathrm{mg}$ cyanidin per $\mathrm{g}$. This result agrees with the findings reported in [5] and its references. However, it was observed that the samples originating from the Netherlands were far richer in monomeric anthocyanins (114.8-369.1 mg/100 g DW) than those from domestic sources (13.3-146.0 mg/100 g DW). A clear trend relating to geographical origin/retail chain was observed in the reference TAC values but not in evidence in TPC values. Whether the VNIR-SWIR and/or ATR-FT-MIR spectroscopic characterization of the samples would expose the same trend is intriguing.

\subsection{VNIR-SWIR Exploratory Approach}

It is accepted that bands at $1415-1512 \mathrm{~nm}, 1650-1750 \mathrm{~nm}$, and $1955-2035 \mathrm{~nm}$ are mainly due to phenolic structure, according to the findings of Dykes et al. [29]. Similarly, in a research study about total anthocyanins in grape juice using NIR spectroscopy, it was found that the spectral range for these phenolic compounds was 1000-1183 nm [30]. Such bands along with those at around $1450 \mathrm{~nm}$ and $1930 \mathrm{~nm}$, corresponding possibly to the $\mathrm{O}-\mathrm{H}$ stretch and $\mathrm{O}-\mathrm{H}$ band combination and the $\mathrm{H}-\mathrm{O}-\mathrm{H}$ deformation combination overtones of hydroxyl groups (e.g., due to water or starch) [16,31] were also evident in the near-infrared spectra of the dry OS samples under study.

As a general rule, the choice of an optimal preprocessing method depends on the characteristics of the dataset and the goal of the analysis [24]. In our study, the VNIR-SWIR spectra of OS samples as the original REF spectral values or as preprocessed spectra are illustrated in Figure 2. Visual assessment of the spectral signatures revealed no significant variation among the dry onion samples. Application of various preprocessing methods resulted in new feature spectral spaces by pronouncing different regions in the VNIRSWIR spectrum and eliminating different effects. SG1 and SG2 emphasize the differences in the visible range and are more prominent to the SWIR region possibly because of greater overlapping of the bands. Similarly, the ABS (including also the SNV values) indicated larger variations than the REF at the first edge of the spectrum in the visible region $(350-750 \mathrm{~nm})$.

In a first exploratory approach to identify diagnostic patterns of sample distribution in the VNIR-SWIR, first derivative of the initial reflectance spectra (SG1) was analyzed via PCA. The analysis resulted in three PCs, the first of which (PC1) accounted for $82.8 \%$ of the total variance, the second (PC2) for $10.3 \%$ and the third (PC3) for $9.6 \%$. The corresponding two-dimensional scoreplots verified that OS samples from domestic sources tended to be clustered separately from those originating from the Netherlands, mainly because of the higher PC2 score values of the latter (Appendix A, Figure A1). This result is quite promising for further modelling of TAC values given our previous observations. No other pattern could be recognized in the sample distribution among the 3-D scoreplot of the PCA model. 


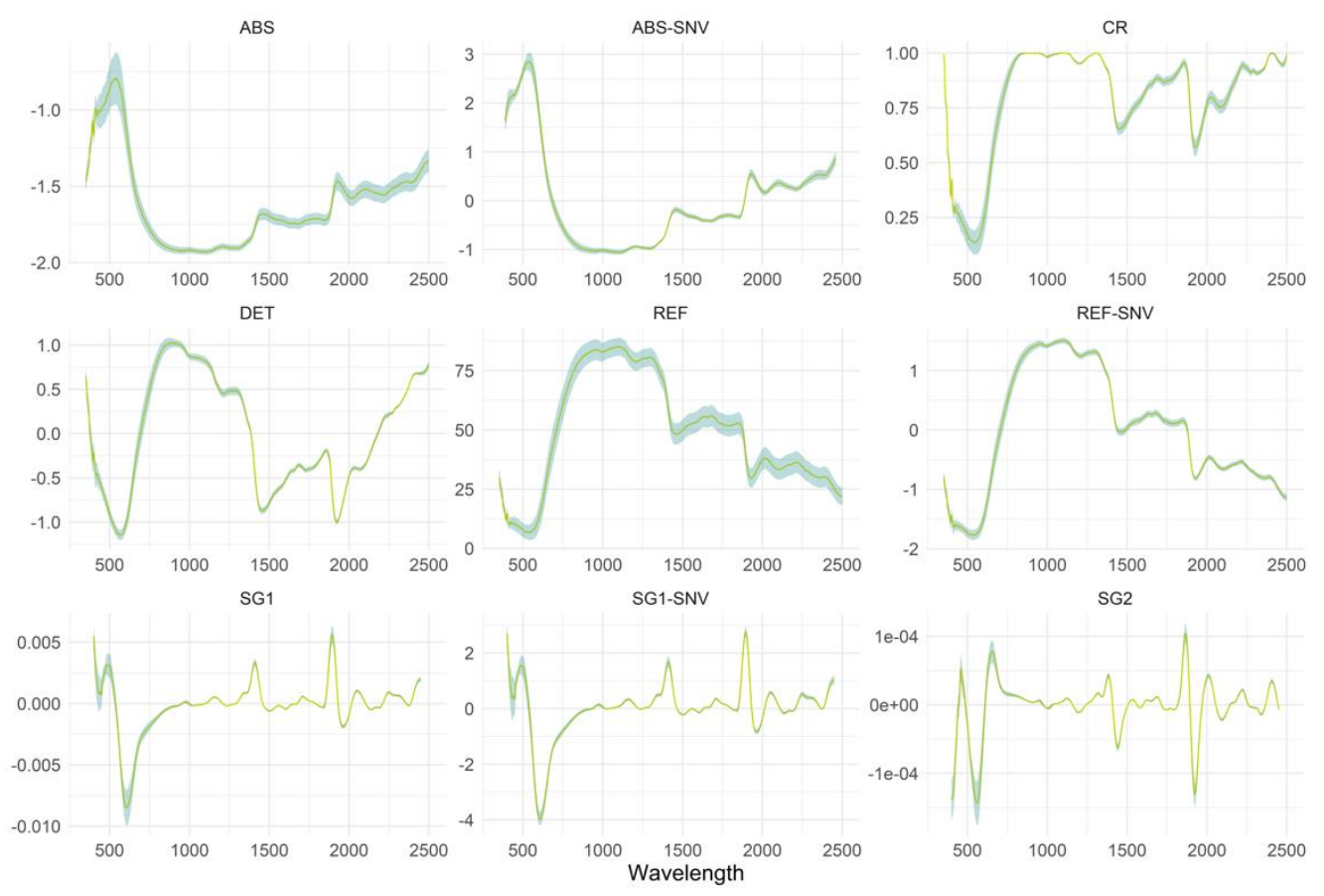

Figure 2. The original and preprocessed VNIR-SWIR spectra of OS samples. The abbreviations of the spectral pre-treatments are described in Section Spectral preprocessing techniques.

\subsection{Monomeric Anthocyanins Prediction Based on the VNIR-SWIR Spectral Datasets}

\subsubsection{Performance of the ML Models}

We first assessed six different ML models in different spectral datasets derived from the various pre-treatments to highlight the impact of the ML techniques in spectroscopic modelling. Overall, the proposed models have a valuable predictive performance $\left(R^{2}\right.$ $>0.80$, and RPIQ $>3$ ). These findings are further illustrated in Figure 3. The results showed that spectral pre-treatments have increased the performance for most of the ML models, with the exception of the DET technique. Notably, modelling of the VNIR-SWIR onion spectral library with the pre-treatment of SG1-SNV and SG2 allowed more accurate predictions of TAC than other preprocessing techniques. A detailed comparison of the model performance obtained with various preprocessing techniques is also provided in Appendix B (Table A2).

We also tested the effectiveness of six ML models by comparing their performance metrics, as shown in Figure 4. In general, better predictive performance was achieved with more complex and supervised algorithms. The k-NN and RF algorithms were found to attain the best performance across all properties. They have enabled more robust predictions $\left(\mathrm{R}^{2}>0.80\right.$, RMSE $<0.53$ and RPIQ $\left.>=3.50\right)$. The results of the various models are reported graphically in Figure 4, in which we can visually compare their performances. The difference with the PLSR algorithm, one of the most commonly applied algorithms in food spectroscopy, is noticeable $\left(\mathrm{R}^{2}=0.81\right.$ and RPIQ $\left.=3.48\right)$, the last having a larger RMSE (0.53). The Cubist and SVM results show lower predictive performance. 


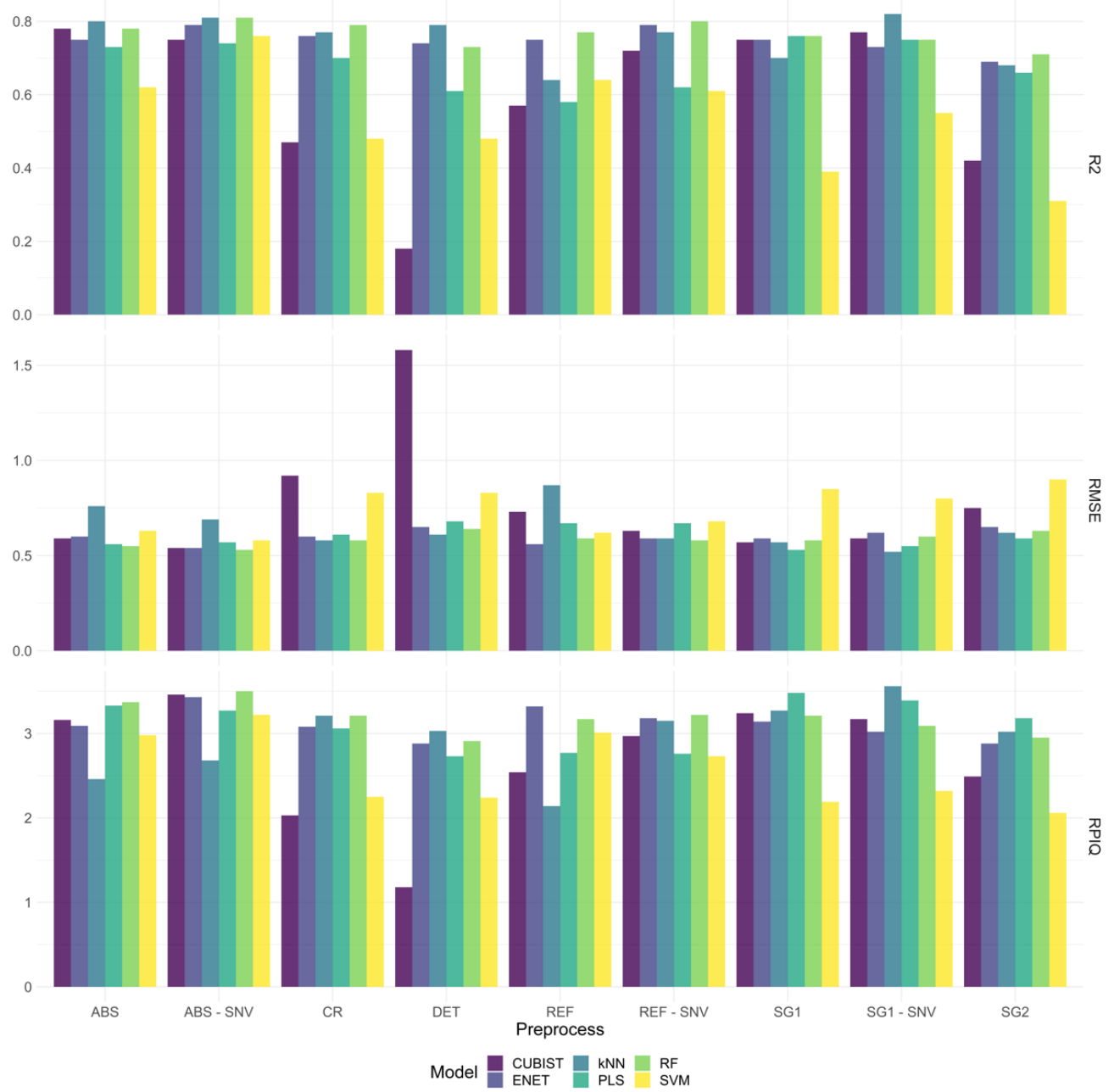

Figure 3. Comparison of the predictive performance metrics of the ML models using the various spectral sources.

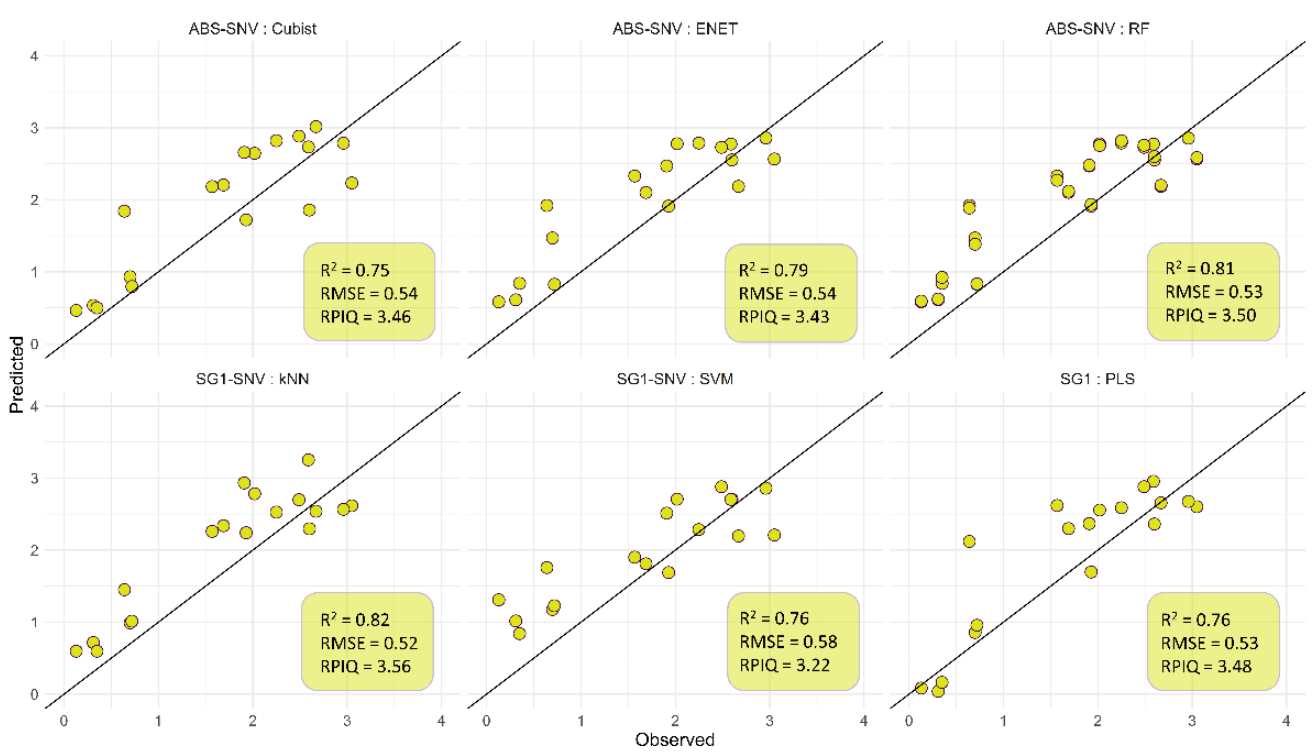

Figure 4. Independent validations set of the anthocyanins predictions from the ML models indicating the observed vs. the predicted values (black solid line represents the 1:1 line). 
It should be highlighted that the selection of the ML model for regression analysis affects the prediction potential of VNIR-SWIR spectral data (Figures 3 and 4). The fact that $\mathrm{k}-\mathrm{NN}$ and RF models presented better performance with smaller prediction errors than wellstudied models in the domain of food spectroscopy (e.g., PLSR and SVM) may be a result of the efficiency of those algorithms to generate subsets with similar characteristics derived by different rules or the distance of the closest neighborhoods. Moreover, it was clearly demonstrated that the various spectral preprocessing techniques result in complementary information that enhances the predictive performance of the ML models compared to those produced with the raw reflectance recordings. Therefore, smoothing (SG1) and/or normalization of the dataset (SNV) should be prioritized in preprocessing steps.

An important aspect of the current study is the interpretability of the underlying models. By visualizing the relative importance of each band across all model-preprocessing combinations, it is possible to recognize those VNIR-SWIR spectral wavelengths that are more prominent in model construction (Figure 5). It is clear that two discrete spectral regions are important; the first one ranges from 400-1000 nm (VNIR), while the second one is in the range $1200-2500 \mathrm{~nm}$ (SWIR). Across nearly all models, VNIR has roughly two main spectral regions: one around $620-650 \mathrm{~nm}$ and the other at the beginning of the spectrum (380-390 nm), depicting, respectively, the characteristic red and purple color of the onion's layers due to the presence of anthocyanins. The finding that the upper SWIR part at $2100-2300 \mathrm{~nm}$ is practically related to aromatic C-H bonds provides valuable information.

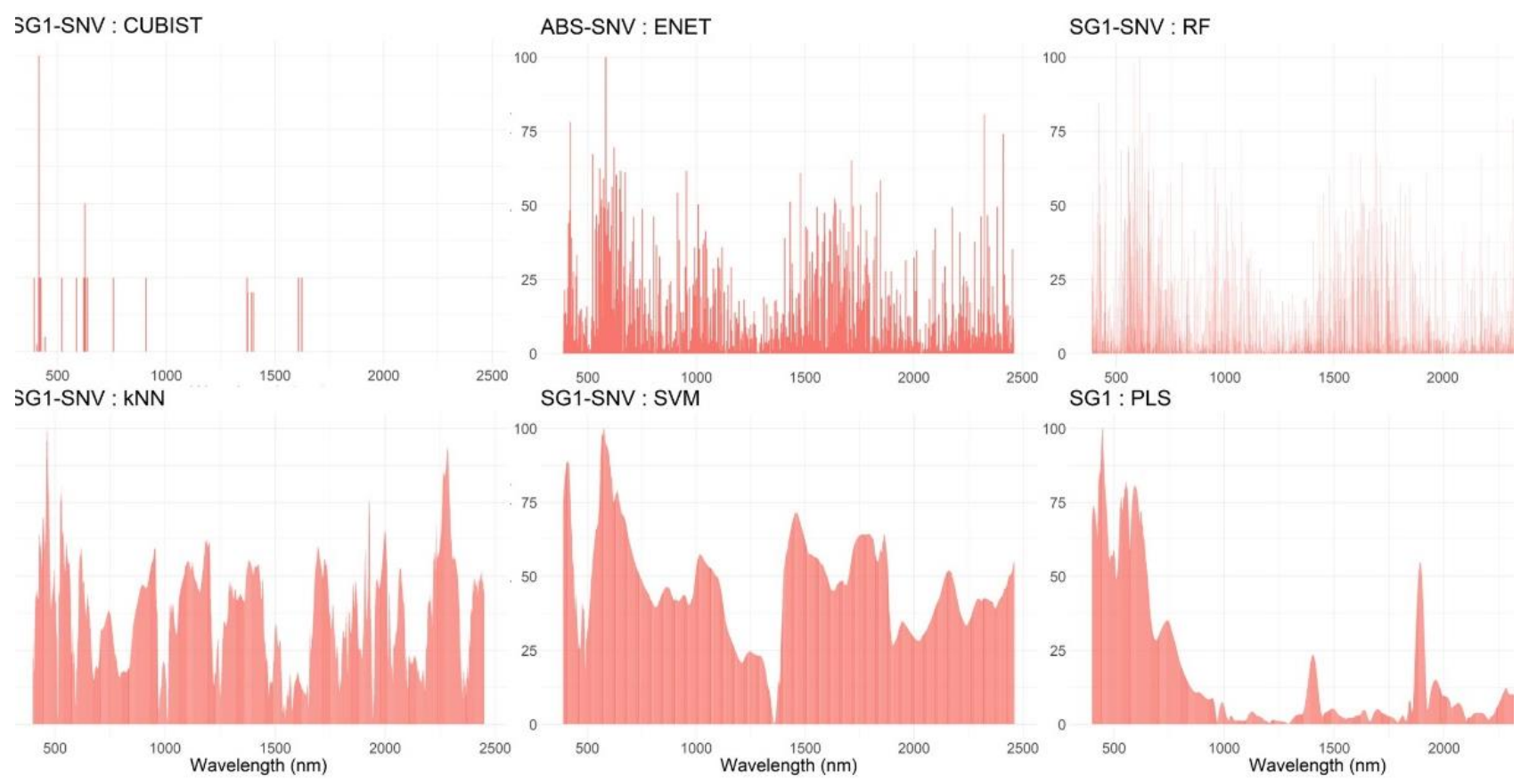

Figure 5. Relative importance of each wavelength in the VNIR-SWIR for the best ML model of TAC values. The spectral datasets used are: SG1-SNV, Savintzky-Golay 1st derivative with standard normal variate, ABS-SNV standard normal variate of absorbances, and SG1 1st derivative of reflectances. The ML algorithms are: ENET, elastic net; RF, random forest; $k$-NN, k-nearest neighbors; SVM, support vector machines for regression; PLS, partial least squares regression and the Cubist algorithm.

\subsubsection{Exploring Shorter Diagnostic Regions in the VNIR-SWIR}

New low-cost spectral devices available in the marketplace interest both researchers and end users to explore the potential of shorter spectral regions for anthocyanin content estimation. However, it is unclear if spectrometers operating solely at VNIR or SWIR could provide sufficient prediction accuracy. Therefore, focus was given to the spectral regions 
between $400-1000 \mathrm{~nm}$ and $1350-2500 \mathrm{~nm}$ because they reflect more clearly spectral regions (as derived from the variable importance analysis) wavelength analysis (see Figure 5).

The $k$-NN model performance (see Figure 4) was tested in two shorter spectral ranges. Then, new rounds of modelling analysis were performed on two sub-sets of the SG1-SNV spectral dataset that corresponded to the selected regions. The results are shown in Table 2. It is clear that accuracy of VNIR-based prediction at $400-1000 \mathrm{~nm}\left(\mathrm{R}^{2}=0.70, \mathrm{RMSE}=0.66\right.$, and RPIQ $=2.80)$ was better than the SWIR-based prediction at $1350-2500 \mathrm{~nm}\left(R^{2}=0.55\right.$, RMSE $=0.75$ and RPIQ $=2.49$ ), but not as high as of that corresponding to the combined spectral region (full spectrum).

Table 2. Predictive performance metrics of the best ML model using the full spectrum and limited spectral regions in VNIR (400-100 nm) and SWIR (1350-2500 nm).

\begin{tabular}{cccc}
\hline Spectral Range & $\mathbf{R}^{\mathbf{2}}$ & RMSE & RPIQ \\
\hline Full spectrum & 0.82 & 0.52 & 3.56 \\
VNIR $(400-1000 \mathrm{~nm})$ & 0.70 & 0.66 & 2.80 \\
SWIR $(1350-2500 \mathrm{~nm})$ & 0.55 & 0.75 & 2.49 \\
\hline
\end{tabular}

Chemometric analysis of the VNIR-SWIR spectra (350 to $2500 \mathrm{~nm}$ ) resulted in satisfactory predictive performance of total anthocyanins content, selectively. The most important features for this purpose were a series of characteristic bands in the visible region of the spectra, mainly at 550-600 $\mathrm{nm}$ (at which these compounds mainly absorb), and in the range close to SWIR (2000-2300 nm) (Figure 5). Future studies could focus on the employment of shorter-range spectroscopic sensors for total anthocyanins in OSW to enable fast, low-cost analyses. This was also proposed recently regarding the evaluation of a MicroElectromechanical systems spectral sensors for soil properties [32]. To computationally enhance the accuracy of prediction, more advanced chemometric approaches can also be employed. They may be combined, (predictions from single ML models developed using bootstrapped samples or the proposed ML algorithms developed via genetic stacking algorithms or even various spectral datasets after pre-processing) instead of relying solely on the best one via novel multi-input deep learning algorithms [33].

\subsection{Identification of Phenolic-Group Diagnostic Bands in the MIR}

The original FT-IR spectra and the accompanying spectral transformation (MSC, second derivative) of OS samples are shown in Figure 6.

In our study of dry OS powder, the characteristic amide-stretching bands of proteins (1550 and $1650 \mathrm{~cm}^{-1}$ ) were not clearly evidenced in the FT-IR spectra. This finding is in line with earlier reports [34]. A weak valley at around $1560 \mathrm{~cm}^{-1}$ revealed in preprocessed spectra signified a possible contribution from nucleic acid bases. Plant cell wall polysaccharides and other types of carbohydrates that are abundant in OS samples (e.g., fructooligosaccharides and pectic oligosaccharides) could be distinguished from some characteristic bands in the region between 1200 and $950 \mathrm{~cm}^{-1}$ [35]. In addition, characteristic bands in the region $1800-1500 \mathrm{~cm}^{-1}$ that are often assigned to esteried and non-esteried carboxyl groups of pectin molecules $[34,35]$ were evidenced in the low frequency region. 

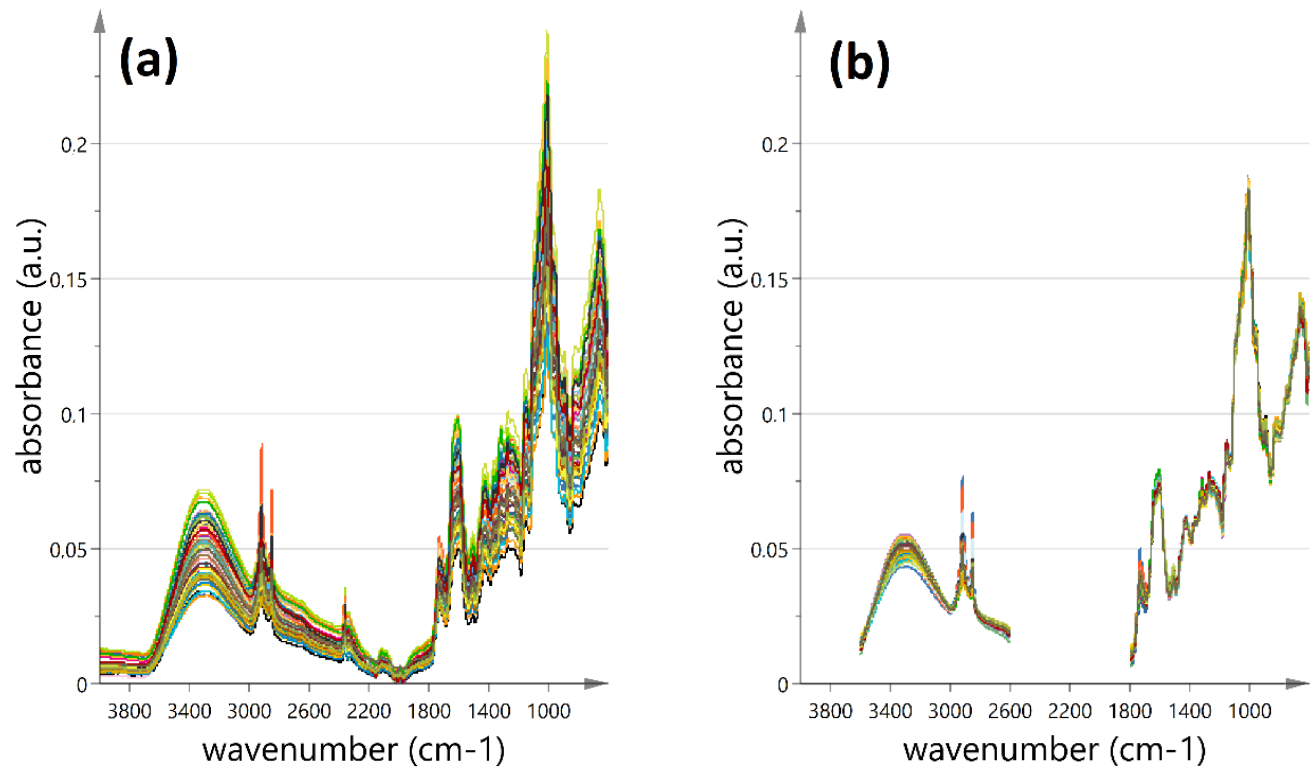

Figure 6. FT-MIR spectra of the dry onion skin samples under study $\left(4000-600 \mathrm{~cm}^{-1}\right)$; (a) spectra without preprocessing; (b) 0th order curves after multi signal correction (MSC).

Table 3 provides an overview of the FT-IR spectral bands that were visually observed as peaks in the original spectra (0th order, after MSC) or as corresponding valleys in the 2nd derivative spectra (2nd order), respectively. The original peaks are clearly much better resolved after 2nd order derivatization of the spectra revealing a number of hidden bands that may carry diagnostic information. This was particularly evidenced in the region below $1000 \mathrm{~cm}^{-1}$ but also between 1400 and $1600 \mathrm{~cm}^{-1}$.

Table 3. Major bands shown as peaks in the 0th order and valleys in the 2nd order derivative FT-MIR spectra of dry OS samples and possible assignment.

\begin{tabular}{|c|c|c|c|c|}
\hline Sample Code & MIR Peaks & MIR Valleys & $\begin{array}{l}\text { Functional Group } \\
\text { Vibrations }\end{array}$ & Possible Identity \\
\hline & 0th Order & 2nd Order & & \\
\hline \multirow{13}{*}{$\begin{array}{l}\text { entry } 1 \\
\text { entry } 2\end{array}$} & 3299.7 & & $\mathrm{v}(\mathrm{O}-\mathrm{H}), \mathrm{v}(\mathrm{N}-\mathrm{H})$ & carbohydrates, water, proteins \\
\hline & 2923.6 & 2917-2918 & $\mathrm{v}_{\text {as }}(\mathrm{C}-\mathrm{H})$ & $-\mathrm{CH} 3$ and $-\mathrm{CH} 2$ - alkanes \\
\hline & 2850.3 & $2848-2850$ & $\mathrm{v}_{\mathrm{as}}(\mathrm{C}-\mathrm{H})$ & $\mathrm{CH} 3$ or $\mathrm{CH} 3-\mathrm{Ar}$ \\
\hline & 1734.7 & $1734-1736$ & $\mathrm{v}(\mathrm{Ar}-\mathrm{C}=\mathrm{O})$ & $\begin{array}{c}\text { aryl carboxylic acid monomers, e.g., hydroxybenzoic } \\
\text { acids }\end{array}$ \\
\hline & & $1717-1718$ & $\mathrm{v}(\mathrm{Ar}-\mathrm{C}=\mathrm{O})$ & aryl ketones, aldehydes \\
\hline & $1637-8$ & $1636-1642$ & $\mathrm{v}(\mathrm{Ar}-\mathrm{C}=\mathrm{O}), \delta(\mathrm{H}-\mathrm{OH})$ & $\begin{array}{l}\text { aryl carboxylic acids and flavonoids, polygalacturonic } \\
\text { acid peptides (amide I), water }\end{array}$ \\
\hline & $1600-2$ & & $\begin{array}{c}\mathrm{v}(\mathrm{C}=\mathrm{C} \text { aromatic }) \\
\mathrm{v}_{\mathrm{as}}(\mathrm{COO}-)\end{array}$ & flavonoids, polygalacturonic acid \\
\hline & & $\begin{array}{c}1561-1562 \\
1521\end{array}$ & $\mathrm{v}(\mathrm{C}=\mathrm{C}$ aromatic $)$ & $\begin{array}{l}\text { flavonoids, nucleic acid ring base } \\
\text { aryl carboxylic acids and flavonoids }\end{array}$ \\
\hline & 1509 & $1503-1507$ & $\delta(\mathrm{C}-\mathrm{H}$ aromatic $)$ & flavonoids \\
\hline & & 1489 & $\delta(\mathrm{C}-\mathrm{H}$ aromatic $)$ & flavonoids, e.g., quercetin and cyanidin glucosides \\
\hline & 1465 & 1471,1464 & $\delta(\mathrm{C}-\mathrm{H}$ aromatic $)$ & aryl carboxylic acids and flavonoids \\
\hline & 1423.2 & 1440,1420 & $\begin{array}{c}\delta(\mathrm{C}-\mathrm{H} \text { aromatic }) \& \\
\mathrm{v}_{\mathrm{S}}(\mathrm{COO}-)\end{array}$ & aryl carboxylic acids, polygalacturonic acid esters \\
\hline & & 1385 & $\delta(\mathrm{O}-\mathrm{H}$ aromatic $)$ & \\
\hline
\end{tabular}


Table 3. Cont.

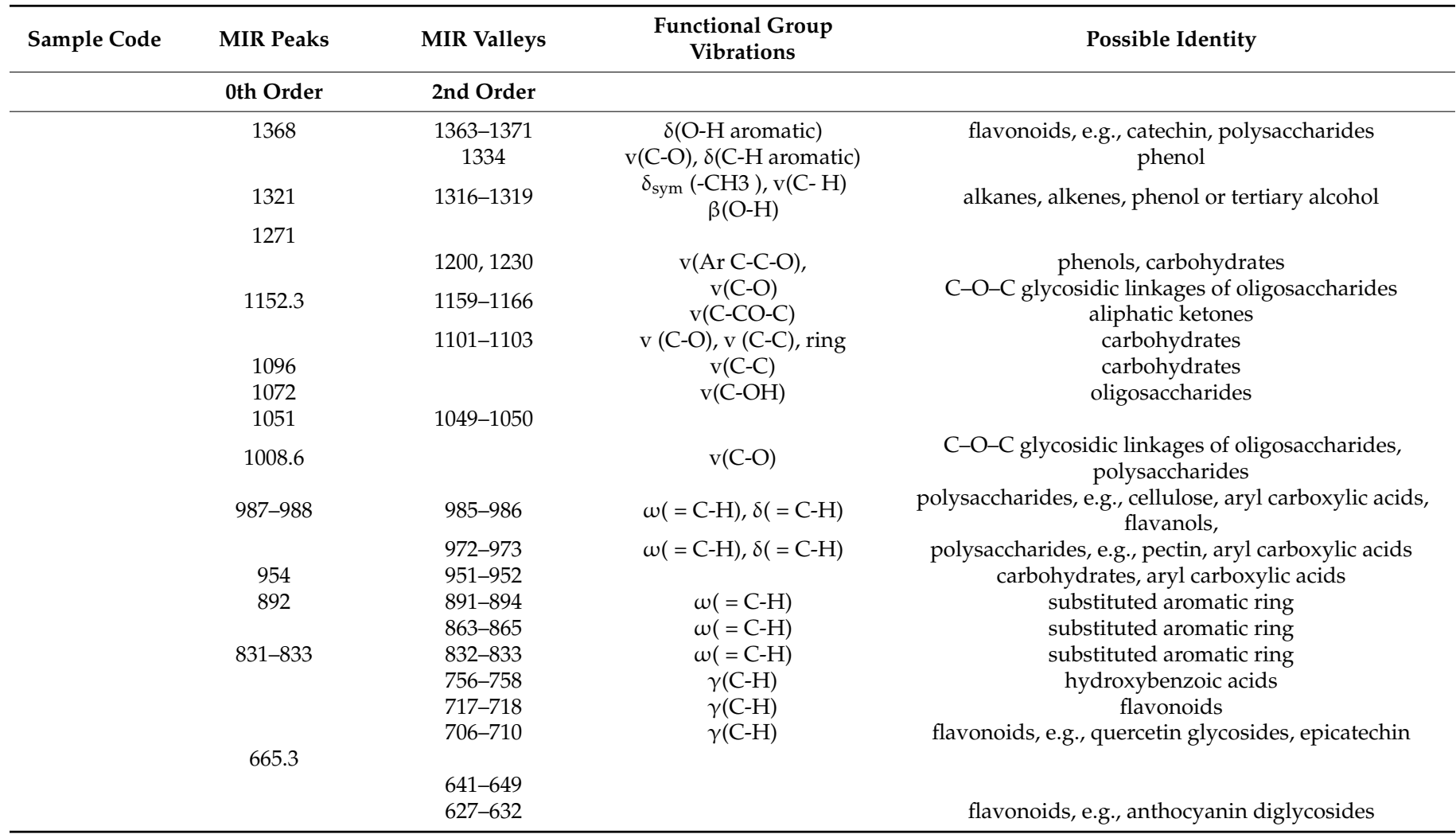

\footnotetext{
${ }^{1}$ Based on $[14,36-38]$. $v$-stretching, as-asymmetric, s-symmetric, $\beta$-in_plane bending, $\gamma$-out-of-plane bending, $\delta$-scissoring or
} deformation, $\varrho$-rocking, $\omega$-wagging.

For phenolic compounds, the two aromatic ring-related bands at around 1600 and $1640 \mathrm{~cm}^{-1}$ were distinct in the spectra of dry OS powder [38]. These two bands were more clearly defined than those at 1185 and $965 \mathrm{~cm}^{-1}$ possibly because of C-O-C and C-OH vibrations of phenols [38]. Given the copresence of polysaccharides and oligosaccharides in the test sample, straightforward assignment of the signals in the latter region is not possible. In a recent study about the potential of FT-IR and PCA to identify individual classes of phenols like flavonols, anthocyanins, and phenolic acids [39], the spectral bands between 1755 and $1400 \mathrm{~cm}^{-1}$ and 1000 and $870 \mathrm{~cm}^{-1}$ were highlighted as the most important. Based on our data, we suggest that stretching vibrations of carboxylic groups at around $1735 \mathrm{~cm}^{-1}$ are more likely assigned to hydroxy-benzoic acid moieties that are present in OS as the major autoxidation products of quercetin. It has been reported that protocatechuic acid and 2-(3,4-dihydroxybenzoyl)-2,4,6-trihydroxybenzofuran-3 (2H)-one are formed during storage of the onions; during that period, enzymatic hydrolysis of quercetin glucosides to release the aglycone form proceeds in parallel with quercetin decomposition reactions [40]. Other conjugates formed due to auto-oxidation may also exist in relatively high amounts [41]. 
To examine further whether these tagged regions of the spectra have diagnostic value related to the total phenolic compound content of OS samples, the spectra of a group of totally 26 OS samples, representing the skin and the 1st inner layer of individual onion bulbs, were acquired and imported to the original dataset. Principal Component Analysis of the data from 0 th and 2nd order derivative spectra in the regions $600-1800 \mathrm{~cm}^{-1}$ resulted in 13 and 6 PCs, respectively. These PCs explained $99.2 \%$ and $75.7 \%$ of the total variance in each case. The analysis of 0th order data extracted eight PCs with very low eigenvalues $(<1)$ that explained almost $8 \%$ of total variance. This result verifies that a considerable amount of variance in the 0th order data is unique or not systematic and is omitted upon 2nd order derivatization. Both rounds of PCA showed that the two groups of samples could be distinguished on the PC1-PC3 scoreplot (Figure 7) on the basis of $\mathrm{t} 3$ score values.
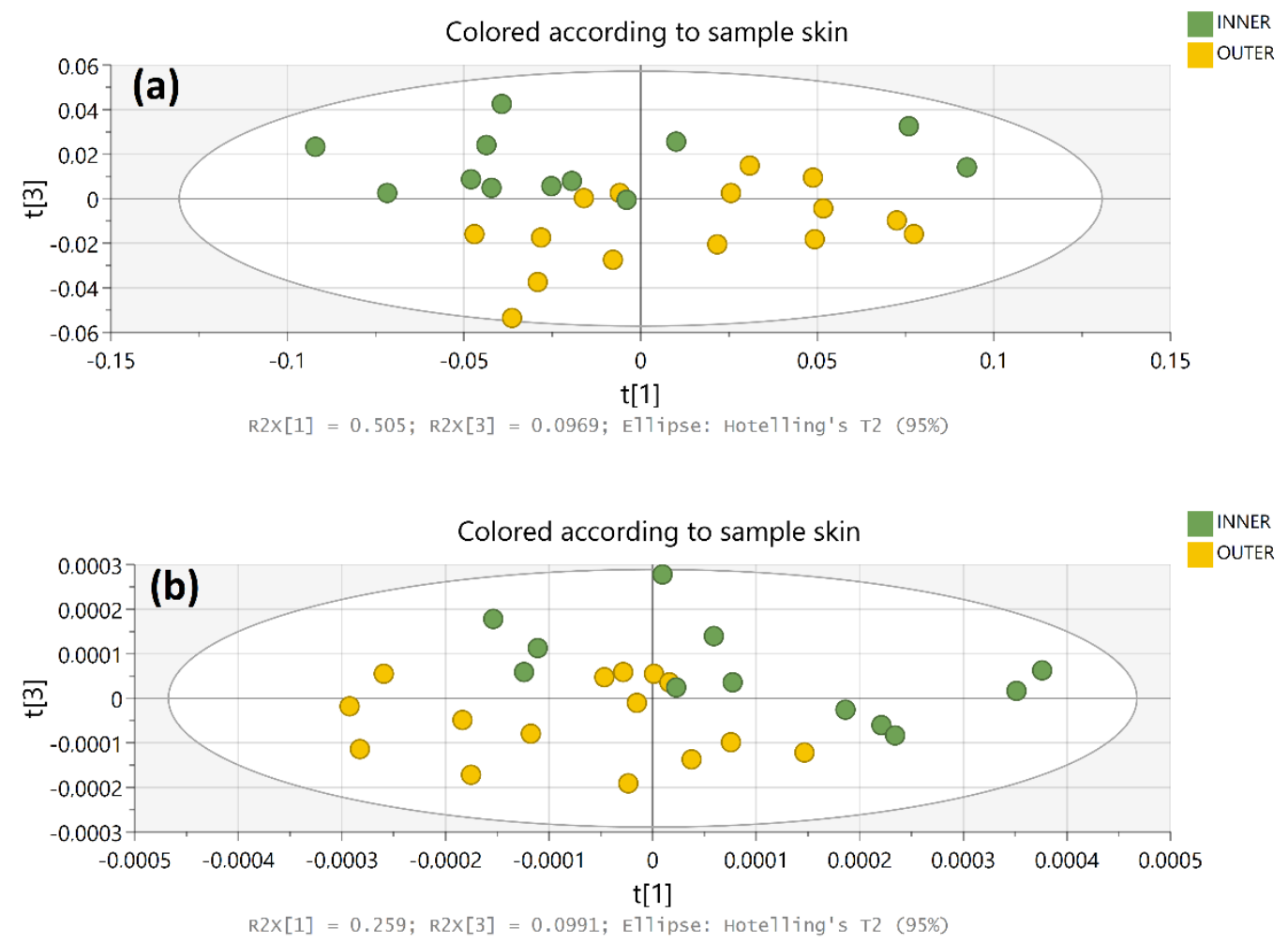

Figure 7. PCA scoreplots of (a) 0th order and (b) 2nd order derivative FT-MIR spectral data (600-1800 $\left.\mathrm{cm}^{-1}\right)$ of dry OS. Different colors indicate outer and 1st inner layer samples from 15 bulbs.

The loading plots of the first and third PCs extracted from each round of PCA are shown in Figure 8. Spectral in the lower frequency region, e.g., at 613, 832, 948, 980 (possibly due to the phenolic ring structure), at $1012-1050 \mathrm{~cm}^{-1}$ (sugars) along with that in the region between 1462-1472, 1500-1520, and at $1734 \mathrm{~cm}^{-1}$ contributed more heavily to the $\mathrm{t} 1-\mathrm{t} 3$ score distribution $(p> \pm 0.6)$ of these OS samples. Special attention was given to the observed variance between 600 and $1000 \mathrm{~cm}^{-1}$ and $1400-1800 \mathrm{~cm}^{-1}$ because it is expected to reflect more clearly differences in the composition of flavonoids and phenolic acid constituents [39]. New rounds of PCA on spectral data that corresponded only to shorter infrared regions revealed that the abundance of carboxylic acid groups remained a distinctive feature of the dry skin and first inner onion bulb layers (Figure 8). Further exclusion of variables between 900 and $1000 \mathrm{~cm}^{-1}$ resulted in similar performance of the PCA model and verified (through corresponding loading plots) that the observed sample allocation is significantly affected by vibrations beyond that region (e.g., ether bonds in carbohydrates). Moreover, it made it possible to highlight that flavonoid ring-related bands around $600-650 \mathrm{~cm}^{-1}$ and $1500-1560 \mathrm{~cm}^{-1}$ are also important for the observed pattern among OS samples. Closer inspection of the FT-MIR spectral curves after 2nd 
order derivatization revealed clear differences in the shape of the bands between 600 and $900 \mathrm{~cm}^{-1}$ that might be partially attributed to skeletal vibrations of different flavylium ring substitution patterns. Considering that anthocyanins constitute a minor percentage of total flavonoids in the skin and outer layers of red onions [6] and the fingerprint region of the spectrum is dominated by highly overlapped signals, it is expected that these shorter-range FT-MIR bands do not assist in quantitative analyses. The FT-MIR data in the specific regions are promising for further exploratory evaluation considering mainly the potential for monitoring oxidation phenomena or other major sources of variance in the phenolic composition, but that is beyond the scope of this study.
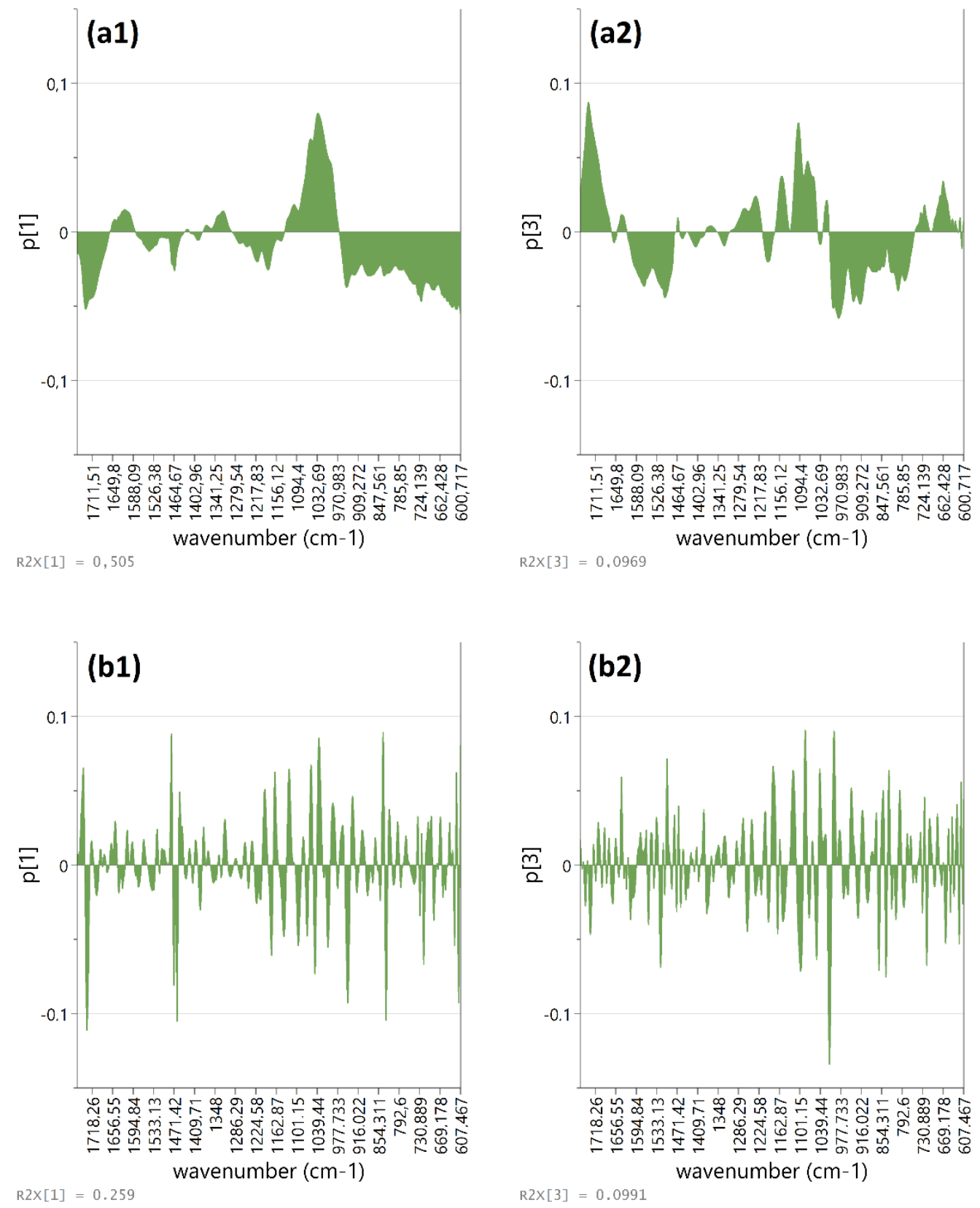

Figure 8. PCA loading plots showing the most important wavenumbers $\left(600-1800 \mathrm{~cm}^{-1}\right)$ from (a) 0th order and (b) 2nd order derivative FT-MIR spectral data of dry OS for the formation of PC1 $(\mathbf{a 1}, \mathbf{b 1})$ and PC3 (a2,b2). 


\section{Conclusions}

Given the heterogeneity of the OSW, which affects their chemical composition, the creation of reference databases is the most essential condition for building robust predictive models. Even though a relatively small OS sample set was used in the current study, the variance in total phenol and total anthocyanin contents of these samples was in the low-high ranges that are typically reported in literature. In the current study, we showed that VNIR-SWIR and FT-IR spectroscopic techniques could be deployed in routine quality control analyses of onion waste, especially in the evaluation of phenolic composition and more particularly in the assessment of the total anthocyanin content. Chemometric analyses of the data through various machine-learning techniques are indispensable for the identification of diagnostic bands across the visible near-to short-wave and mid-infrared regions. Above all, a k-NN model of 1st derivative spectra in the region of 350-2500 nm was the most powerful for the prediction of the monomeric anthocyanin content in dry red OS samples $\left(R^{2}=0.82\right.$, RMSE $=0.52$, and RPIQ $\left.=3.56\right)$. The performance of the predictive model remained satisfactory when it was assessed in a shorter, more selective spectral range. This result supports the perspective for the potential uses of low-cost spectroscopic sensors in this field. The FT-IR spectral fingerprint was more informative about the inherent quality characteristics of OSW as it enables structural assignments. Overall, we suggest that non-destructive spectroscopic tools operating in the visible-near-short-wave and midinfrared regions can be employed in real-time quality control of OSW if the spectral data are of high quality and well-demonstrated diagnostic value or predictive accuracy with regard to the audit target, e.g., anthocyanins content. Updating the reference onion skin spectral libraries and evaluation of the model performance are, thus, in progress.

Author Contributions: Conceptualization, N.T., S.A.O., G.Z. and I.M.; methodology, N.T., S.A.O., A.T.; software, K.K., S.A.O.; validation, N.T., S.A.O.; investigation, S.A.O. and A.T.; data curation, N.T., A.T. and S.A.O.; writing-original draft preparation, N.T., S.A.O., I.M.; writing-review and editing, S.A.O., G.Z., I.M.; visualization, N.T.; supervision, I.M. All authors have read and agreed to the published version of the manuscript.

Funding: This research received no external funding.

Institutional Review Board Statement: Not applicable.

Informed Consent Statement: Not applicable.

Data Availability Statement: The data presented in this study are available on request from the corresponding author (GM).

Acknowledgments: Authors would like to thank Interdisciplinary Agri-Food Center (KEAGRO), Aristotle University of Thessaloniki for providing the access to the equipment of the unit.

Conflicts of Interest: The authors declare no conflict of interest.

\section{Appendix A}

An appropriate tuning of hyperparameters ensures the ML models' consistency. Thus, a grid search on a five-fold cross-validation experiment was conducted to select the optimal hyperparameters for ML model. The optimal set of hyper-parameters for each ML algorithm is presented in Table 1. 
Table 1. Optimal hyper-parameters of ML models, selected by the grid search with the cross-validation approach.

\begin{tabular}{|c|c|c|c|c|c|c|c|c|c|c|}
\hline \multirow{2}{*}{ Pre-Treatment Methods } & \multirow{2}{*}{$\begin{array}{l}\text { PLS } \\
\text { LVs }\end{array}$} & \multicolumn{2}{|l|}{ RF } & \multicolumn{2}{|c|}{ Cubist } & \multicolumn{2}{|c|}{ ENET } & \multirow{2}{*}{$\begin{array}{c}\text { k-NN } \\
k\end{array}$} & \multicolumn{2}{|c|}{ SVM } \\
\hline & & mtry & ntree & $\mathrm{C}$ & $\mathbf{n}$ & $\mathbf{s}$ & $\lambda 2$ & & $\mathrm{C}$ & sigma \\
\hline ABS & 3 & 9 & 200 & 50 & 0 & 0.75 & 0.010 & 8 & 1 & 0.001 \\
\hline ABS-SNV & 2 & 4 & 250 & 10 & 0 & 0.75 & 0.010 & 12 & 1 & 0.001 \\
\hline $\mathrm{CR}$ & 2 & 19 & 150 & 100 & 9 & 0.75 & 0.005 & 6 & 1 & 0.001 \\
\hline DET & 2 & 3 & 150 & 100 & 0 & 0.50 & 0.005 & 8 & 1 & 0.001 \\
\hline REF & 2 & 20 & 150 & 1 & 0 & 0.75 & 0.010 & 13 & 1 & 0.001 \\
\hline REF-SNV & 3 & 17 & 200 & 10 & 0 & 0.50 & 0.010 & 5 & 1 & 0.001 \\
\hline SG1 & 2 & 13 & 250 & 10 & 5 & 0.50 & 0.010 & 6 & 1 & 0.001 \\
\hline SG1-SNV & 2 & 2 & 150 & 50 & 0 & 0.50 & 0.010 & 3 & 1 & 0.001 \\
\hline SG2 & 2 & 20 & 150 & 1 & 5 & 0.50 & 0.010 & 3 & 1 & 0.001 \\
\hline
\end{tabular}

(a)

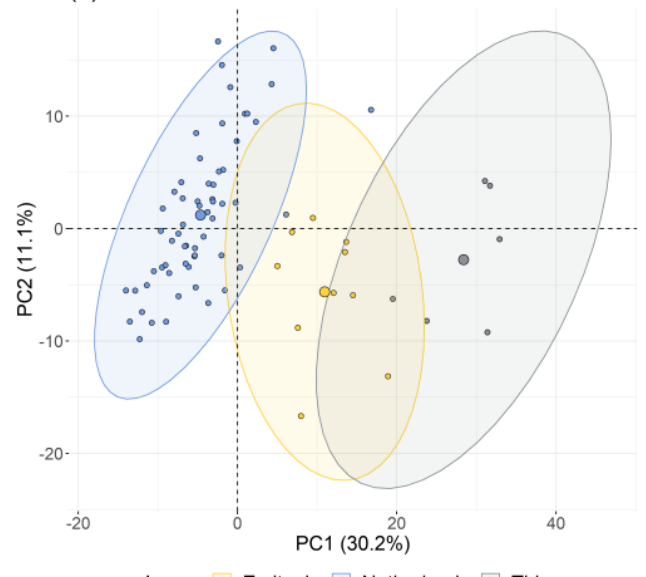

(b)

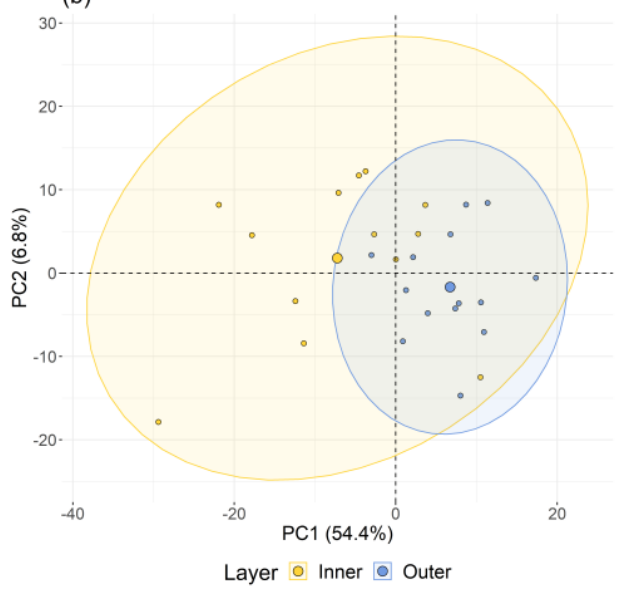

Figure A1. Scatter diagrams (a) of the first vs second principal components extracted from PCA of VNIR-SWIR spectral data of dry onion skin indicating the source of supply; and (b) of the first vs third principal components extracted from PCA of VNIR-SWIR spectral data of dry onion skin indicating the layer under study. 


\section{Appendix B}

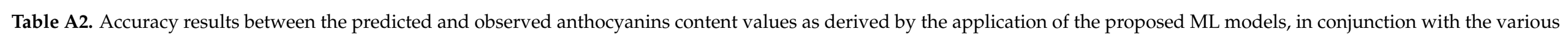
pre-processing methods.

\begin{tabular}{|c|c|c|c|c|c|c|c|c|c|c|c|c|c|c|c|c|c|c|}
\hline \multirow[t]{2}{*}{$\begin{array}{l}\text { Pre-Processing } \\
\text { Techniques }\end{array}$} & \multicolumn{3}{|c|}{ PLS } & \multicolumn{3}{|c|}{$\mathbf{R F}$} & \multicolumn{3}{|c|}{ CUBIST } & \multicolumn{3}{|c|}{ ENET } & \multicolumn{3}{|c|}{ K-NN } & \multicolumn{3}{|c|}{ SVM } \\
\hline & $\mathbf{R}^{2}$ & RMSE & RPIQ & $\mathbf{R}^{2}$ & RMSE & RPIQ & $\mathbf{R}^{2}$ & RMSE & RPIQ & $\mathbf{R}^{2}$ & RMSE & RPIQ & $\mathbf{R}^{2}$ & RMSE & RPIQ & $\mathbf{R}^{2}$ & RMSE & RPIQ \\
\hline ABS & 0.57 & 0.67 & 2.76 & 0.76 & 0.58 & 3.17 & 0.57 & 0.73 & 2.54 & 0.75 & 0.56 & 3.32 & 0.64 & 0.87 & 2.13 & 0.63 & 0.61 & 3.01 \\
\hline ABS-SNV & 0.73 & 0.55 & 3.32 & 0.78 & 0.55 & 3.37 & 0.78 & 0.58 & 3.15 & 0.74 & 0.60 & 3.09 & 0.79 & 0.75 & 2.46 & 0.62 & 0.62 & 2.97 \\
\hline CR & 0.76 & 0.53 & 3.48 & 0.76 & 0.57 & 3.21 & 0.74 & 0.57 & 3.24 & 0.75 & 0.59 & 3.14 & 0.69 & 0.56 & 3.27 & 0.38 & 0.84 & 2.19 \\
\hline DET & 0.66 & 0.58 & 3.17 & 0.70 & 0.63 & 2.94 & 0.42 & 0.74 & 2.49 & 0.69 & 0.64 & 2.87 & 0.65 & 0.61 & 3.03 & 0.31 & 0.90 & 2.05 \\
\hline REF & 0.69 & 0.60 & 3.06 & 0.78 & 0.57 & 3.20 & 0.46 & 0.91 & 2.02 & 0.75 & 0.60 & 3.07 & 0.77 & 0.57 & 3.20 & 0.48 & 0.82 & 2.25 \\
\hline REF-SNV & 0.62 & 0.67 & 2.76 & 0.80 & 0.57 & 3.22 & 0.72 & 0.62 & 2.97 & 0.79 & 0.58 & 3.17 & 0.76 & 0.59 & 3.14 & 0.60 & 0.68 & 2.72 \\
\hline SG1 & 0.60 & 0.68 & 2.72 & 0.73 & 0.63 & 2.91 & 0.18 & 1.58 & 1.17 & 0.74 & 0.64 & 2.87 & 0.79 & 0.61 & 3.02 & 0.48 & 0.83 & 2.23 \\
\hline SG1-SNV & 0.74 & 0.54 & 3.39 & 0.75 & 0.60 & 3.08 & 0.77 & 0.58 & 3.16 & 0.73 & 0.61 & 3.02 & 0.81 & 0.52 & 3.56 & 0.54 & 0.80 & 2.31 \\
\hline SG2 & 0.74 & 0.56 & 3.27 & 0.81 & 0.53 & 3.49 & 0.74 & 0.53 & 3.46 & 0.79 & 0.54 & 3.43 & 0.81 & 0.69 & 2.68 & 0.76 & 0.57 & 3.22 \\
\hline
\end{tabular}




\section{References}

1. Sharma, K.; Mahato, N.; Nile, S.H.; Lee, E.T.; Lee, Y.R. Economical and environmentally-friendly approaches for usage of onion (Allium cepa L.) waste. Food Funct. 2016, 7, 3354-3369. [CrossRef]

2. FAO Production/Yield Quantities of Onions, Dry in Europe. Available online: http://www.fao.org/faostat/en/\#data/QC/ visualize (accessed on 9 June 2021).

3. Benítez, V.; Mollá, E.; Martín-Cabrejas, M.A.; Aguilera, Y.; López-Andréu, F.J.; Cools, K.; Terry, L.A.; Esteban, R.M. Characterization of Industrial Onion Wastes (Allium cepa L.): Dietary Fibre and Bioactive Compounds. Plant Foods Hum. Nutr. 2011, 66, 48-57. [CrossRef]

4. Celano, R.; Docimo, T.; Piccinelli, A.L.; Gazzerro, P.; Tucci, M.; Di Sanzo, R.; Carabetta, S.; Campone, L.; Russo, M.; Rastrelli, L.; et al. Onion Peel: Turning a Food Waste into a Resource. Antioxidants 2021, 10, 304. [CrossRef]

5. Albishi, T.; John, J.A.; Al-Khalifa, A.S.; Shahidi, F. Antioxidative phenolic constituents of skins of onion varieties and their activities. J. Funct. Foods 2013, 5, 1191-1203. [CrossRef]

6. Rhodes, M.J.C.; Price, K.R. Analytical problems in the study of flavonoid compounds in onions. Food Chem. 1996, 57, 113-117. [CrossRef]

7. Mourtzinos, I.; Prodromidis, P.; Grigorakis, S.; Makris, D.P.; Biliaderis, C.G.; Moschakis, T. Natural food colorants derived from onion wastes: Application in a yoghurt product. Electrophoresis 2018, 39, 1975-1983. [CrossRef]

8. Santiago, B.; Arias Calvo, A.; Gullón, B.; Feijoo, G.; Moreira, M.T.; González-García, S. Production of flavonol quercetin and fructooligosaccharides from onion (Allium cepa L.) waste: An environmental life cycle approach. Chem. Eng. J. 2020, $392,123772$. [CrossRef]

9. Kimoto-Nira, H.; Ohashi, Y.; Amamiya, M.; Moriya, N.; Ohmori, H.; Sekiyama, Y. Fermentation of onion (Allium cepa L.) peel by lactic acid bacteria for production of functional food. J. Food Meas. Charact. 2020, 14, 142-149. [CrossRef]

10. Patra, J.K.; Kwon, Y.; Baek, K.H. Green biosynthesis of gold nanoparticles by onion peel extract: Synthesis, characterization and biological activities. Adv. Powder Technol. 2016, 27, 2204-2213. [CrossRef]

11. Imbert, E. Food waste valorization options: Opportunities from the bioeconomy. Open Agric. 2017, 2, 195-204. [CrossRef]

12. Mustafa, F.; Andreescu, S. Chemical and biological sensors for food-quality monitoring and smart packaging. Foods 2018, 7, 168. [CrossRef] [PubMed]

13. Tsakanikas, P.; Karnavas, A.; Panagou, E.Z.; Nychas, G.J. A machine learning workflow for raw food spectroscopic classification in a future industry. Sci. Rep. 2020, 10, 11212. [CrossRef]

14. Lu, X.; Wang, J.; Al-Qadiri, H.M.; Ross, C.F.; Powers, J.R.; Tang, J.; Rasco, B.A. Determination of total phenolic content and antioxidant capacity of onion (Allium cepa) and shallot (Allium oschaninii) using infrared spectroscopy. Food Chem. 2011, 129, 637-644. [CrossRef] [PubMed]

15. Vincke, D.; Baeten, V.; Sinnaeve, G.; Dardenne, P.; Pierna, J.A.F. Determination of Outer Skin in Dry Onions by Hyperspectral Imaging Spectroscopy and Chemometrics. NIR News 2014, 25, 9-12. [CrossRef]

16. Wang, W.; Li, C.; Gitaitis, R.D. Optical properties of healthy and diseased onion tissues in the visible and near-infrared spectral region. Trans. ASABE 2014, 57, 1771-1782. [CrossRef]

17. Lee, J.; Durst, R.W.; Wrolstad, R.E. Determination of total monomeric anthocyanin pigment content of fruit juices, beverages, natural colorants, and wines by the $\mathrm{pH}$ differential method: Collaborative study. J. AOAC Int. 2005, 88, 1269-1278. [CrossRef]

18. Rinnan, Å.; van den Berg, F.; Engelsen, S.B. Review of the most common pre-processing techniques for near-infrared spectra. TrAC Trends Anal. Chem. 2009, 28, 1201-1222. [CrossRef]

19. Minasny, B.; McBratney, A.B. A conditioned Latin hypercube method for sampling in the presence of ancillary information. Comput. Geosci. 2006, 32, 1378-1388. [CrossRef]

20. Geladi, P.; Kowalski, B.R. Partial least-squares regression: A tutorial. Anal. Chim. Acta 1986. [CrossRef]

21. Svetnik, V.; Liaw, A.; Tong, C.; Christopher Culberson, J.; Sheridan, R.P.; Feuston, B.P. Random Forest: A Classification and Regression Tool for Compound Classification and QSAR Modeling. J. Chem. Inf. Comput. Sci. 2003. [CrossRef]

22. Quinlan, J.R. Learning with continuous classes. In Proceedings of the Australian Joint Conference on Artificial Intelligence, Hobart, Australia, 16-18 November 1992; pp. 343-348.

23. Drucker, H.; Surges, C.J.C.; Kaufman, L.; Smola, A.; Vapnik, V. Support vector regression machines. In Proceedings of the Advances in Neural Information Processing Systems; MIT Press: Cambridge, MA, USA, 1997; pp. 155-161.

24. Engel, J.; Gerretzen, J.; Szymańska, E.; Jansen, J.J.; Downey, G.; Blanchet, L.; Buydens, L.M.C. Breaking with trends in preprocessing? TrAC Trends Anal. Chem. 2013, 50, 96-106. [CrossRef]

25. Ordoudi, S.A.; de los Mozos Pascual, M.; Tsimidou, M.Z. On the quality control of traded saffron by means of transmission Fourier-transform mid-infrared (FT-MIR) spectroscopy and chemometrics. Food Chem. 2014, 150, 414-421. [CrossRef] [PubMed]

26. R Development Core Team. R: A Language and Environment for Statistical Computing; R Found. Stat. Comput.: Vienna, Austria, 2013.

27. Kuhn, M. Building Predictive Models in R Using the caret Package. J. Stat. Softw. Artic. 2008, 28, 1-26. [CrossRef]

28. Kiassos, E.; Mylonaki, S.; Makris, D.P.; Kefalas, P. Implementation of response surface methodology to optimise extraction of onion (Allium cepa) solid waste phenolics. Innov. Food Sci. Emerg. Technol. 2009. [CrossRef]

29. Dykes, L.; Hoffmann, L.; Portillo-Rodriguez, O.; Rooney, W.L.; Rooney, L.W. Prediction of total phenols, condensed tannins, and 3-deoxyanthocyanidins in sorghum grain using near-infrared (NIR) spectroscopy. J. Cereal Sci. 2014, 60, 138-142. [CrossRef] 
30. Caramês, E.T.S.; Alamar, P.D.; Poppi, R.J.; Pallone, J.A.L. Rapid Assessment of Total Phenolic and Anthocyanin Contents in Grape Juice Using Infrared Spectroscopy and Multivariate Calibration. Food Anal. Methods 2017, 10, 1609-1615. [CrossRef]

31. Lohumi, S.; Lee, S.; Lee, W.H.; Kim, M.S.; Mo, C.; Bae, H.; Cho, B.K. Detection of starch adulteration in onion powder by FT-NIR and FT-IR spectroscopy. J. Agric. Food Chem. 2014, 62, 9246-9251. [CrossRef]

32. Tang, Y.; Jones, E.; Minasny, B. Evaluating low-cost portable near infrared sensors for rapid analysis of soils from South Eastern Australia. Geoderma Reg. 2020, 20, e00240. [CrossRef]

33. Tsakiridis, N.L.; Keramaris, K.D.; Theocharis, J.B.; Zalidis, G.C. Simultaneous prediction of soil properties from VNIR-SWIR spectra using a localized multi-channel 1-D convolutional neural network. Geoderma 2020, 367, 114208. [CrossRef]

34. McCann, M.C.; Hammouri, M.; Wilson, R.; Belton, P.; Roberts, K. Fourier transform infrared microspectroscopy is a new way to look at plant cell walls. Plant Physiol. 1992, 100, 1940-1947. [CrossRef] [PubMed]

35. Sun, D.-W. Infrared Spectroscopy for Food Quality Analysis and Control, 1st ed.; Sun, D.-W., Ed.; Elsevier Inc.: New York, NY, USA, 2009; ISBN 9780123741363.

36. Socrates, G. Infrared and Raman Characteristic Group Frequencies, 3rd ed.; Socrates, G., Ed.; John Wiley and Sons, Ltd.: Chichester, UK, 2004; Volume 35, ISBN 978-0-470-09307-8.

37. Lu, X.; Ross, C.F.; Powers, J.R.; Rasco, B.A. Determination of quercetins in onion (Allium cepa) using infrared spectroscopy. J. Agric. Food Chem. 2011, 59, 6376-6382. [CrossRef] [PubMed]

38. Schulz, H.; Baranska, M. Identification and quantification of valuable plant substances by IR and Raman spectroscopy. Vib. Spectrosc. 2007, 43, 13-25. [CrossRef]

39. Abbas, O.; Compère, G.; Larondelle, Y.; Pompeu, D.; Rogez, H.; Baeten, V. Phenolic compound explorer: A mid-infrared spectroscopy database. Vib. Spectrosc. 2017, 92, 111-118. [CrossRef]

40. Khiari, Z.; Makris, D.P. Stability and transformation of major flavonols in onion (Allium cepa) solid wastes. J. Food Sci. Technol. 2012, 49, 489-494. [CrossRef]

41. Tram, N.L.; Hazama, C.; Shimoyamada, M.; Ando, H.; Kato, K.; Yamauchi, R. Antioxidative compounds from the outer scales of onion. J. Agric. Food Chem. 2005, 53, 8183-8189. [CrossRef] 